\title{
Global Well-Posedness and Stability for a Viscoelastic Plate Equation with a Time Delay
}

\author{
Baowei Feng \\ College of Economic Mathematics, Southwestern University of Finance and Economics, Chengdu 611130, China \\ Correspondence should be addressed to Baowei Feng; bwfeng@swufe.edu.cn
}

Received 21 January 2015; Revised 18 March 2015; Accepted 18 March 2015

Academic Editor: Ivanka Stamova

Copyright (C) 2015 Baowei Feng. This is an open access article distributed under the Creative Commons Attribution License, which permits unrestricted use, distribution, and reproduction in any medium, provided the original work is properly cited.

A plate equation with a memory term and a time delay term in the internal feedback is investigated. Under suitable assumptions, we establish the global well-posedness of the initial and boundary value problem by using the Faedo-Galerkin approximations and some energy estimates. Moreover, by using energy perturbation method, we prove a general decay result of the energy provided that the weight of the delay is less than the weight of the damping.

\section{Introduction}

In this paper, we are concerned with the following plate equation with a memory term and a time delay term in the internal feedback:

$$
\begin{aligned}
u_{t t}+ & \Delta^{2} u-M\left(\|\nabla u\|^{2}\right) \Delta u \\
& +\int_{0}^{t} g(t-s) \Delta u(s) d s+\mu_{1} u_{t}(x, t) \\
& +\mu_{2} u_{t}(x, t-\tau)+f(u)=0,
\end{aligned}
$$

where $\Omega \subseteq \mathbb{R}^{n}(n \geq 1)$ is a bounded domain with smooth boundary $\partial \Omega$. Here $M(\cdot)$ is a function satisfying suitable conditions (see below), $\mu_{1}, \mu_{2}$ are positive constants, and $\tau$ > 0 represents the time delay.

Equation (1) with the memory term $\int_{0}^{t} g(t-s) \Delta u(s) d s$, where the function $g$ is called kernel, can be regarded as a fourth order viscoelastic plate equation with a lower order perturbation, and it can be also regarded as an elastoplastic flow equation with some kind of memory effect.

In this paper, we consider the following initial conditions:

$$
\begin{aligned}
& u(x, 0)=u_{0}(x), \quad u_{t}(x, 0)=u_{1}(x), \quad x \in \Omega, \\
& u_{t}(x, t-\tau)=h_{0}(x, t-\tau), \quad x \in \Omega, t \in(0, \tau)
\end{aligned}
$$

and the following boundary conditions:

$$
u=\Delta u=0, \quad \text { on } \partial \Omega \times \mathbb{R}^{+} .
$$

Fourth order equations with lower order perturbation are related to models of elastoplastic microstructure flows. For the single plate equation without delay, that is, $\mu_{2}=0$, as considered by Woinowsky-Krieger [1], the author first proposed the one-dimensional nonlinear equation of vibration of beams, which is given by

$$
u_{t t}+\alpha u_{x x x x}-\left(\beta+\gamma \int_{0}^{L}\left|u_{x}\right|^{2} d x\right) u_{x x}=0,
$$

where $L$ is the length of the beam and $\alpha, \beta, \gamma$ are positive physical constants. The nonlinear part of (4) represents for the extensible effect for the beam whose ends are restrained to remain in a fixed distance apart in its transverse vibrations. A more general equation of (4) reads

$$
u_{t t}-M\left(\|\nabla u\|^{2}\right) \Delta u+\Delta^{2} u+g\left(u_{t}\right)+f(u)=h(x),
$$

where $M(\cdot)$ is a function satisfying some conditions. There are so many existing results concerning global existence, stability, and long-time dynamics for (5); we would like to refer the reader to de Brito [2], Cavalcanti et al. [3, 4], Ma [5], Ma and Narciso [6], de Lacerda Oliveira and de Lima [7], J. Y. Park and S. H. Park [8], Patcheu [9], Rivera [10, 11], 
Tusnal [12], Vasconcellos and Teixeira [13], Yang [14, 15], and the references therein. Very recently, Andrade et al. [16] investigated a viscoelastic plate equation with $p$-Laplacian and memory terms with strong damping

$$
u_{t t}+\Delta^{2} u-\Delta_{p} u+\int_{0}^{t} g(t-s) \Delta u(s) d s-\Delta u_{t}+f(u)=0
$$

where $\Delta_{p} u=\operatorname{div}\left(|\nabla u|^{p-2} \nabla u\right)$ is the $p$-Laplacian operator. Under suitable assumptions on the memory kernel $g$ and a forcing term $f$, the authors proved the existence of weak solutions by using Faedo-Galerkin approximations, the uniqueness of strong solutions, and the exponential stability of solutions to (6) with initial and boundary value problem. For more results on viscoelastic equations, we can refer to Berrimi and Messaoudi [17], Messaoudi [18], Messaoudi and Tartar $[19,20]$, and the references therein.

In recent years, many mathematical workers studied some systems with time delay effects. Datko et al. [21] studied the following system:

$$
\begin{gathered}
u_{t t}-\Delta u=0, \quad x \in \Omega, t>0, \\
u(x, t)=0, \quad x \in \Gamma_{0}, t>0, \\
\frac{\partial u}{\partial \nu}=\mu_{1} u_{t}(x, t)+\mu_{2} u_{t}(x, t-\tau), \quad x \in \Gamma_{1}, t>0, \\
u(x, 0)=u_{0}(x), \quad u_{t}(x, 0)=u_{1}(x), \quad x \in \Omega, \\
u(x, t-\tau)=g_{0}(x, t-\tau), \quad x \in \Omega, t \in[0, \tau] .
\end{gathered}
$$

By using an observability inequality, they proved the exponential stability for the energy when $\mu_{2}<\mu_{1}$. Subsequently, $\mathrm{Xu}$ et al. [22] obtained the same result as in [21] for the one space dimension by using the spectral analysis approach. Later on, Kirane and Said-Houari [23] considered a viscoelastic wave equation with a delay term in internal feedback with initial conditions and boundary value conditions of Dirichlet type. Under suitable assumptions on the relaxation function and some restriction on the parameters $\mu_{1}$ and $\mu_{2}$, they established the global well-posedness of the system. Moreover, under the assumption $\mu_{2} \leq \mu_{1}$ between the weight of the delay term in the feedback and the weight of the term without delay, the authors proved a general decay of the total energy of the system. For more some results concerning the different boundary conditions under an appropriate assumption between $\mu_{1}$ and $\mu_{2}$, one can refer to Nicaise and Pignotti [24], Nicaise et al. [25], Nicaise and Valein [26], and the references therein.

Equation (1) is a plate equation with a memory term and a time delay term in the internal feedback. Noting that $\mu_{1} \neq$ 0 , we know that it is a plate equation with weak damping. For viscoelastic plate equations, it is well known that one considered a memory of the form $\int_{0}^{t} g(t-s) \Delta^{2} u(s) d s$ (see, e.g., $[10,27,28])$. However, because the main dissipation of the system (1)-(3) is given by a weak damping $u_{t}$, here we consider a weaker memory, acting only on $\Delta u$. To the best of our knowledge, the global well-posedness and energy decay for system (1)-(3) were not previously considered. So the objective of this work is to establish the global well-posedness and stability of initial boundary value problem (1)-(3). The main dissipation of the system (1)-(3) is given by a weak damping $u_{t}$, which makes the analysis in this work different from [16], because the authors considered the case of a strong damping $-\Delta u_{t}$ in $[16]$.

The outline of this paper is as follows. In Section 2, we give some preparations for our consideration and our main results. In Section 3, we establish the global posedness of the system by using the Faedo-Galerkin approximations and some energy estimates. In Section 4, we will show a general decay result of the energy by using energy perturbation method provided that the weight of the delay is less than the weight of the damping.

The notation in this paper will be as follows: $L^{q}, 1 \leq q \leq$ $+\infty, W^{m, q}, m \in \mathbb{N}, H^{1}=W^{1,2}, H_{0}^{1}=W_{0}^{1,2}$ denote the usual (Sobolev) spaces on $\Omega$. In addition, $\|\cdot\|_{B}$ denotes the norm in the space $B$, and we also put $\|\cdot\|=\|\cdot\|_{L^{2}(\Omega)}$.

\section{Preliminaries and Main Results}

In this section, we give some preparations for our consideration and our main results.

(i) We assume that $M(\cdot): \mathbb{R}^{+} \rightarrow \mathbb{R}^{+}$is a $C^{1}$ function satisfying

$$
\begin{gathered}
z M(z) \geq \widehat{M}(z), \\
\widehat{M}(z)=\int_{0}^{z} M(s) d s
\end{gathered}
$$

(if $M(z)$ is monotone nondecreasing).

(ii) For the memory kernel $g(t)$, we assume that

$$
\begin{gathered}
(\mathrm{G} 1) g: \mathbb{R}^{+} \rightarrow \mathbb{R}^{+} \text {is a function satisfying } \\
g(t) \in C^{1}\left(\mathbb{R}^{+}\right) \cap L^{1}\left(\mathbb{R}^{+}\right), \quad g(0)>0, \\
1-\lambda \int_{0}^{\infty} g(s) d s=l>0,
\end{gathered}
$$

where $\lambda>0$ is the embedding constant for $\|\nabla u\|^{2} \leq \lambda\|\Delta u\|^{2}$.

(G2) There exists a positive nonincreasing differentiable function $\mu(t)$ such that

$$
\begin{gathered}
g^{\prime}(t) \leq-\mu(t) g(t), \quad \forall t \geq 0, \\
\int_{0}^{\infty} \mu(t) d t=\infty .
\end{gathered}
$$

(iii) The nonlinear term $f(u)$ satisfies

$$
\begin{array}{r}
f(0)=0, \quad|f(u)-f(v)| \leq c\left(1+|u|^{\rho}+|v|^{\rho}\right)|u-v|, \\
\forall u, v \in \mathbb{R},
\end{array}
$$


where $c>0$ is a constant, and $\rho$ satisfies

$$
0<\rho<\frac{4}{n-4} \quad \text { if } \geq 5, \quad \rho>0 \quad \text { if } 1 \leq n \leq 4 .
$$

We denote $\widehat{f}(z)=\int_{0}^{z} f(s) d s$ and assume that

$$
0 \leq \widehat{f}(u) \leq f(u) u, \quad \forall u \in \mathbb{R} .
$$

In order to deal with the delay feedback term, motivated by $[24,26]$, we introduce the following new dependent variable:

$$
z(x, \rho, t)=u_{t}(x, t-\tau \rho), \quad x \in \Omega, \rho \in(0,1), t>0 .
$$

Then it is easy to verify

$$
\tau z_{t}(x, \rho, t)+z_{\rho}(x, \rho, t)=0, \quad \text { in } \Omega \times(0,1) \times(0, \infty) .
$$

Thus, problem (1)-(3) is transformed into

$$
\begin{gathered}
u_{t t}(x, t)+\Delta^{2} u-M\left(\|\nabla u\|^{2}\right) \Delta u \\
+\int_{0}^{t} g(t-s) \Delta u(s) d s+\mu_{1} u_{t}+\mu_{2} z(x, 1, t)=0 \\
\tau z_{t}(x, \rho, t)+z_{\rho}(x, \rho, t)=0
\end{gathered}
$$

with $x \in \Omega, \rho \in(0,1)$ and $t>0$, and the initial and boundary conditions are

$$
\begin{gathered}
u(x, 0)=u_{0}, \quad u_{t}(x, 0)=u_{1}, \quad x \in \Omega, \\
z(x, \rho, 0)=h_{0}(x, t-\tau), \quad(x, t) \in \Omega \times(0, \tau), \\
u=\Delta u=0, \quad \text { on } \partial \Omega \times \mathbb{R}^{+}, \\
z(x, 0, t)=u_{t}(x, t) \quad x \in \Omega, t>0 .
\end{gathered}
$$

Let $\xi$ be a positive constant satisfying

$$
\tau \mu_{2}<\xi<\tau\left(2 \mu_{1}-\mu_{2}\right) .
$$

Now we define the weak solutions of (1)-(3): for given initial data $\left(u_{0}, u_{1}\right) \in H^{2}(\Omega) \cap H_{0}^{1}(\Omega) \times L^{2}(\Omega)$, we say that a function $z=\left(u, u_{t}\right) \in C\left(\mathbb{R}^{+}, H^{2}(\Omega) \cap H_{0}^{1}(\Omega) \times L^{2}(\Omega)\right)$ is a weak solution to the problem (1)-(3) if $z(0)=\left(u_{0}, u_{1}\right)$ and

$$
\begin{aligned}
& \left(u_{t t}, \omega\right)+(\Delta u, \Delta \omega)+\left(M\left(\|\nabla u\|^{2}\right) \nabla u, \nabla \omega\right) \\
& \quad-\int_{0}^{t} g(t-s)(\nabla u(s), \nabla \omega) d s \\
& \quad+\mu_{1}\left(u_{t}, \omega\right)+\mu_{2}\left(u_{t}(t-\tau), \omega\right)+(f(u), \omega)=0,
\end{aligned}
$$

for all $\omega \in H^{2}(\Omega) \cap H_{0}^{1}(\Omega)$.

Next we state the global well-posedness of problem (17)(18) given in the following theorem.

Theorem 1. Let $\mu_{2} \leq \mu_{1}$ hold and assume the assumptions (8)(14) hold. (i) If the initial data $\left(u_{0}, u_{1}\right) \in\left(H^{2}(\Omega) \cap H_{0}^{1}(\Omega) \times L^{2}(\Omega)\right)$, $h_{0} \in L^{2}(\Omega \times(0,1))$, then problem (17)-(18) has a weak solution such that

$u \in C\left(\mathbb{R}^{+} ; H^{2}(\Omega) \cap H_{0}^{1}(\Omega)\right) \cap C^{1}\left(\mathbb{R}^{+} ; L^{2}(\Omega)\right)$,

$u_{t} \in L^{2}\left(\mathbb{R}^{+} ; L^{2}(\Omega)\right)$

(ii) If the initial data $\left(u_{0}, u_{1}\right) \in H_{\partial \Omega}^{3}(\Omega) \times H_{0}^{1}(\Omega), h_{0} \in$ $H^{1}(\Omega \times(0,1))$, where

$$
H_{\partial \Omega}^{3}(\Omega)=\left\{u \in H^{3}(\Omega) \mid u=\Delta u=0 \text { on } \partial \Omega\right\} \text {, }
$$

then the above weak solution has higher regularity

$$
\begin{aligned}
& u \in L^{\infty}\left(\mathbb{R}^{+}, H^{3}(\Omega)\right), \\
& u_{t} \in L^{\infty}\left(\mathbb{R}^{+}, H_{0}^{1}(\Omega)\right) \cap L^{2}\left(\mathbb{R}^{+}, H_{0}^{1}(\Omega)\right) .
\end{aligned}
$$

(iii) In both cases, we have that the solution $\left(u, u_{t}\right)$ depends continuously on the initial data in $H^{2}(\Omega) \cap H_{0}^{1}(\Omega) \times$ $L^{2}(\Omega)$. In particular, problem (17)-(18) has a unique weak solution.

We define the energy of problem (17)-(18) by

$$
\begin{aligned}
E(t)= & \frac{1}{2}\left\|u_{t}(t)\right\|^{2}+\frac{1}{2}\|\Delta u(t)\|^{2}+\frac{1}{2} \widehat{M}\left(\|\nabla u(t)\|^{2}\right) \\
& +\int_{\Omega} \widehat{f}(u(t)) d x+\frac{\xi}{2} \int_{\Omega} \int_{0}^{1} z^{2}(x, \rho, t) d \rho d x
\end{aligned}
$$

Finally, we give the energy decay of problem (17)-(18).

Theorem 2. Let $\mu_{2}<\mu_{1}$ hold and assume the assumptions (8)(14) hold. In both cases (i) and (ii), there exist two constants $\alpha>0$ and $\beta>0$ such that the energy $E(t)$ defined by (24) satisfies

$$
E(t) \leq \alpha \exp \left(-\beta \int_{0}^{t} \mu(s) d s\right), \quad \forall t \geq 0
$$

\section{The Global Well-Posedness}

In this section, we will prove the global existence and the uniqueness of the solution of problem (17)-(18) by using the classical Faedo-Galerkin approximations along with some priori estimates. We only prove the existence of solution in (i). For the existence of stronger solution in (ii), we can use the same method as in (i) and one can refer to Andrade e al. [16] and Jorge Silva and Ma [28].

3.1. Approximate Problem. Let $\left\{w_{j}\right\}$ be the Galerkin basis given by the eigenfunctions of $\Delta^{2}$ with boundary condition $u=\Delta u=0$ on $\partial \Omega$. For any $m \geq 1$, let $W_{m}=\operatorname{span}\left\{w_{1}, w_{2}\right.$, $\left.\ldots, w_{n}\right\}$.

We define for $1 \leq j \leq m$ the sequence $\phi_{j}(x, \rho)$ by

$$
\phi_{j}(x, 0)=w_{j}(x) .
$$


Then we can extend $\phi_{j}(x, 0)$ by $\phi_{j}(x, \rho)$ over $L^{2}(\Omega \times(0,1))$ and denote $V_{m}=\operatorname{span}\left\{\phi_{1}, \phi_{2}, \ldots, \phi_{n}\right\}$.

Given initial data $u_{0} \in H^{2}(\Omega) \cap H_{0}^{1}(\Omega), u_{1} \in L^{2}(\Omega)$, and $h_{0} \in L^{2}(\Omega \times(0,1))$, we define the approximations

$$
\begin{gathered}
u_{m}(t)=\sum_{j=1}^{m} g_{j m}(t) w_{j}(x), \\
z_{m}(x, \rho, t)=\sum_{j=1}^{m} h_{j m}(t) \phi_{j}(x, \rho),
\end{gathered}
$$

which satisfy the following approximate problem:

$$
\begin{gathered}
\left(u_{m t t}(t), w_{j}\right)+\left(\Delta u_{m}(t), \Delta w_{j}\right)+\left(-M\left(\left\|\nabla u_{m}\right\|^{2}\right) \Delta u_{m}, w_{j}\right) \\
+\left(f\left(u_{m}(t)\right), w_{j}\right)+\int_{0}^{t} g(t-s)\left(\Delta u_{m}(s), w_{j}\right) d s \\
+\left(\mu_{1} u_{m t}(t), w_{j}\right)+\left(\mu_{2} z_{m}(x, 1, t), w_{j}\right)=0, \\
\left(\tau z_{m t}(x, \rho, t), \phi_{j}\right)+\left(z_{m \rho}(x, \rho, t), \phi_{j}\right)=0,
\end{gathered}
$$

with initial conditions

$$
u_{m}(0)=u_{0}^{m}, \quad u_{m t}(0)=u_{1}^{m} \quad z_{m}(x, \rho, 0)=z_{0}^{m},
$$

which satisfies

$$
\begin{aligned}
& u_{0}^{m} \longrightarrow u_{0} \quad \text { strongly in } H^{2}(\Omega) \cap H_{0}^{1}(\Omega), \\
& u_{1}^{m} \longrightarrow u_{1} \quad \text { strongly in } L^{2}(\Omega), \\
& z_{0}^{m} \longrightarrow h_{0} \quad \text { strongly in } L^{2}(\Omega \times(0,1)) .
\end{aligned}
$$

By using standard ordinary differential equations theory, the problem (28)-(29) has a solution $\left(g_{j m}, h_{j m}\right)_{j=1, m}$ defined on $\left[0, t_{m}\right)$. The following estimate will give the local solution being extended to $[0, T]$, for any given $T>0$.

3.2. A Priori Estimate. Now multiplying the first approximate equation of (28) by $g_{j m}^{\prime}$, we see that

$$
\begin{aligned}
& \frac{d}{d t}\left(\frac{1}{2}\left\|u_{m t}(t)\right\|^{2}+\frac{1}{2}\left\|\Delta u_{m}(t)\right\|^{2}+\frac{1}{2} \widehat{M}\left(\left\|\nabla u_{m}(t)\right\|^{2}\right)\right. \\
& \left.\quad+\int_{\Omega} \widehat{f}\left(u_{m}(t)\right)\right)+\mu_{1}\left\|u_{n t}(t)\right\|^{2} \\
& \quad+\mu_{2} \int_{\Omega} z_{m}(x, 1, t) u_{m t}(t) d x \\
& \quad-\int_{0}^{t} g(t-s)\left(\nabla u_{m}(s), \nabla u_{m t}(t)\right) d s=0 .
\end{aligned}
$$

Noting the following fact:

$$
\begin{gathered}
\frac{1}{2} \frac{d}{d t}\left((g \circ \nabla u)(t)-\int_{0}^{t} g(s) d s \cdot\|\nabla u(t)\|^{2}\right) \\
+\int_{0}^{t} \int_{0}^{t} g(t-s)\left(\nabla u(s), \nabla u_{t}(t)\right) d s \\
=\frac{1}{2}\left(g^{\prime} \circ \nabla u\right)(t)-\frac{1}{2} g(t)\|\nabla u(t)\|^{2},
\end{gathered}
$$

where

$$
(g \circ \nabla u)(t)=\int_{0}^{t} g(t-s)\|\nabla u(s)-\nabla u(t)\|^{2} d s,
$$

we know that

$$
\begin{gathered}
\frac{1}{2} \frac{d}{d t}\left(\left\|u_{m t}(t)\right\|^{2}+\left\|\Delta u_{m}(t)\right\|^{2}+\widehat{M}\left(\left\|\nabla u_{m}(t)\right\|^{2}\right)\right. \\
-\left(\int_{0}^{t} g(s) d s\right)\left\|\nabla u_{m}(t)\right\|^{2} \\
\left.+2 \int_{\Omega} \widehat{f}\left(u_{m}(t)\right) d x+\left(g \circ \nabla u_{m}\right)(t)\right) \\
+\mu_{1}\left\|u_{m t}(t)\right\|^{2}+\mu_{2} \int_{\Omega} z_{m}(x, 1, t) u_{m t}(t) d x \\
=\frac{1}{2}\left(g^{\prime} \circ \nabla u_{m}\right)(t)-\frac{1}{2} g(t)\left\|\nabla u_{m}(t)\right\|^{2} .
\end{gathered}
$$

Multiplying the second approximate equation of (28) by $(\xi / \tau) h_{j m}^{\prime}$ and then integrating over $(0, t) \times(0,1)$, we obtain

$$
\begin{aligned}
\frac{\xi}{2} \int_{\Omega} & \int_{0}^{1} z_{m}^{2}(x, \rho, t) d \rho d x \\
& +\frac{\xi}{\tau} \int_{0}^{t} \int_{\Omega} \int_{0}^{1} z_{m \rho} z_{m}(x, \rho, s) d \rho d x d s \\
= & \frac{\xi}{2} \int_{\Omega} \int_{0}^{1} z_{0}^{m}(x, \rho) d \rho d x .
\end{aligned}
$$

A straightforward calculation gives

$$
\begin{aligned}
& \int_{0}^{t} \int_{\Omega} \int_{0}^{1} z_{m \rho} z_{m}(x, \rho, s) d \rho d x d s \\
& \quad= \frac{1}{2} \int_{0}^{t} \int_{\Omega} \int_{0}^{1} \frac{\partial}{\partial \rho} z_{m}^{2}(x, \rho, s) d \rho d x d s \\
& \quad=\frac{1}{2} \int_{0}^{t} \int_{\Omega}\left(z_{m}^{2}(x, 1, s)-z_{m}^{2}(x, 0, s)\right) d x d s .
\end{aligned}
$$

Now integrating (34) and using (35)-(36) and $z_{m}^{2}(x, 0, s)=$ $u_{m t}^{2}(s)$, we infer that

$$
\begin{aligned}
\mathscr{E}_{m}( & t)+\left(\mu_{1}-\frac{\xi}{2 \tau}\right) \int_{0}^{t}\left\|u_{m t}(t)\right\|^{2} d s \\
+ & \frac{\xi}{2 \tau} \int_{0}^{t} \int_{\Omega} z_{m}^{2}(x, 1, s) d x d s \\
& +\mu_{2} \int_{0}^{t} \int_{\Omega} z_{m}(x, 1, s) u_{m t}(s) d x d s \\
& +\frac{1}{2} \int_{0}^{t} g(s)\left\|\nabla u_{m}(s)\right\|^{2} d s \\
& -\frac{1}{2} \int_{0}^{t}\left(g^{\prime} \circ \nabla u_{m}\right)(s) d s=\mathscr{E}_{m}(0),
\end{aligned}
$$


with

$$
\begin{aligned}
\mathscr{E}_{m}(t)=\frac{1}{2}\left(\left\|u_{m t}(t)\right\|^{2}+\left\|\Delta u_{m}(t)\right\|^{2}+\widehat{M}\left(\left\|\nabla u_{m}(t)\right\|^{2}\right)\right. \\
-\left(\int_{0}^{t} g(s) d s\right)\left\|\nabla u_{m}(t)\right\|^{2} \\
\left.\quad+2 \int_{\Omega} \widehat{f}\left(u_{m}(t)\right) d x+\left(g \circ \nabla u_{m}\right)(t)\right) \\
+\frac{\xi}{2} \int_{\Omega} \int_{0}^{1} z_{m}^{2}(x, \rho, t) d \rho d x .
\end{aligned}
$$

Then we have the following cases.

(i) Consider $\mu_{2}<\mu_{1}$. Using Young's inequality, we have

$$
\begin{gathered}
\mu_{2} \int_{0}^{t} \int_{\Omega} z_{m}(x, 1, s) u_{m t}(s) d x d s \\
\geq-\frac{\mu_{2}}{2} \int_{0}^{t} \int_{\Omega} z_{m}^{2}(x, 1, s) d x d s \\
\quad-\frac{\mu_{2}}{2} \int_{0}^{t}\left\|u_{m t}^{2}(s)\right\| d s,
\end{gathered}
$$

which, together with (37), yields

$$
\begin{aligned}
\mathscr{E}_{m}(t) & +\left(\mu_{1}-\frac{\xi}{2 \tau}-\frac{\mu_{2}}{2}\right) \int_{0}^{t}\left\|u_{m t}(t)\right\|^{2} d s \\
& +\left(\frac{\xi}{2 \tau}-\frac{\mu_{2}}{2}\right) \int_{0}^{t} \int_{\Omega} z_{m}^{2}(x, 1, s) d x d s \\
& +\frac{1}{2} \int_{0}^{t} g(s)\left\|\nabla u_{m}(s)\right\|^{2} d s \\
& -\frac{1}{2} \int_{0}^{t}\left(g^{\prime} \circ \nabla u_{m}\right)(s) d s \leq \mathscr{E}_{m}(0) .
\end{aligned}
$$

It follows from (19) that there exist two constants $c_{1}>0$ and $c_{2}>0$ such that

$$
\begin{aligned}
\mathscr{E}_{m}(t) & +c_{1} \int_{0}^{t}\left\|u_{m t}(t)\right\|^{2} d s \\
& +c_{2} \int_{0}^{t} \int_{\Omega} z_{m}^{2}(x, 1, s) d x d s \\
& +\frac{1}{2} \int_{0}^{t} g(s)\left\|\nabla u_{m}(s)\right\|^{2} d s \\
& -\frac{1}{2} \int_{0}^{t}\left(g^{\prime} \circ \nabla u_{m}\right)(s) d s \leq \mathscr{E}_{m}(0) .
\end{aligned}
$$

(ii) Consider $\mu_{1}=\mu_{2}$. Taking $\xi=\tau \mu_{1}=\tau \mu_{2}$ and using (37), we know that

$$
\begin{aligned}
\mathscr{E}_{m}(t) & +\frac{1}{2} \int_{0}^{t} g(s)\left\|\nabla u_{m}(s)\right\|^{2} d s \\
- & \frac{1}{2} \int_{0}^{t}\left(g^{\prime} \circ \nabla u_{m}\right)(s) d s \leq \mathscr{E}_{m}(0) .
\end{aligned}
$$

Then, in both cases, we infer that there exists a positive constant $C$ independent on $m$ such that

$$
\mathscr{E}_{m}(t) \leq C, \quad t \geq 0 .
$$

It follows from (9), (14), and (43) that

$$
\begin{gathered}
\left\|u_{m t}(t)\right\|^{2}+\left\|\Delta u_{m}(t)\right\|^{2}+\widehat{M}\left(\left\|\nabla u_{m}(t)\right\|^{2}\right) \\
+\int_{\Omega} \int_{0}^{1} z_{m}^{2}(x, \rho, t) d \rho d x \leq C .
\end{gathered}
$$

Thus we can obtain $t_{m}=T$, for all $T>0$.

3.3. Passage to Limit. From (44), we conclude that for any $m \in$ $\mathbb{N}$,

$$
\begin{aligned}
& u_{m} \text { is bounded in } L^{\infty}\left(\mathbb{R}^{+} ; H^{2}(\Omega) \cap H_{0}^{1}(\Omega)\right), \\
& u_{m t} \text { is bounded in } L^{\infty}\left(\mathbb{R}^{+} ; L^{2}(\Omega)\right), \\
& z_{m} \text { is bounded in } L^{\infty}\left(\mathbb{R}^{+} ; L^{2}(\Omega \times(0,1))\right) .
\end{aligned}
$$

Thus we get

$u_{m} \rightarrow u \quad$ weakly star in $L^{\infty}\left(\mathbb{R}^{+} ; H^{2}(\Omega) \cap H_{0}^{1}(\Omega)\right)$,

$u_{m t} \rightarrow u_{t} \quad$ weakly star in $L^{2}\left(\mathbb{R}^{+} ; L^{2}(\Omega)\right)$,

$z_{m} \rightarrow z \quad$ weakly star in $L^{2}\left(\mathbb{R}^{+} ; L^{2}(\Omega \times(0,1))\right)$.

By (45)-(47), we can also deduce that $u_{m}$ is bounded in $L^{2}\left(\mathbb{R}^{+} ; H^{2}(\Omega) \cap H_{0}^{1}(\Omega)\right)$ and $u_{m t}$ is bounded in $L^{2}\left(\mathbb{R}^{+} ; L^{2}(\Omega)\right)$. Then from Aubin-Lions theorem [29], we infer that for any $T>0$,

$$
u_{m} \longrightarrow u \text { strongly in } L^{\infty}\left(0, T ; H_{0}^{1}(\Omega)\right) .
$$

We also obtain by Lemma 1.4 in Kim [30] that

$$
u_{m} \longrightarrow u \quad \text { strongly in } C\left(0, T ; H_{0}^{1}(\Omega)\right) .
$$

Then we can pass to limit the approximate problem (28)-(29) in order to get a weak solution of problem (17)-(18).

3.4. Continuous Dependence and Uniqueness. Firstly we prove the continuous dependence and uniqueness for stronger solutions of problem (17)-(18).

Let $\left(u(t), u_{t}(t), z_{1}(x, \rho, t)\right)$ and $\left(v(t), v_{t}(t), z_{2}(x, \rho, t)\right)$ be two global solutions of problem (17)-(18) with respect to initial data $\left(u_{0}, u_{1}, h_{01}\right)$ and $\left(v_{0}, v_{1}, h_{02}\right)$ respectively. Let $\omega(t)=u(t)-v(t), \chi(x, \rho, t)=z_{1}(x, \rho, t)-z_{2}(x, \rho, t)$. Then $(\omega(t), \chi(x, \rho, t))$ verifies

$$
\begin{gathered}
\omega_{t t}+\Delta^{2} \omega-\left(M\left(\|\nabla u\|^{2}\right) \Delta u-M\left(\|\nabla v\|^{2}\right) \Delta v\right) \\
+\int_{0}^{t} g(t-s) \Delta \omega(s) d s+\mu_{1} \omega_{t} \\
+\mu_{2} \chi(x, 1, t)+f(u)-f(v)=0, \\
\tau \chi_{t}+\chi_{\rho}=0,
\end{gathered}
$$


with boundary conditions

$$
\omega=\Delta \omega=0, \quad \text { on } \partial \Omega
$$

and initial data

$$
\begin{gathered}
\omega(x, 0)=\omega_{0}, \quad \omega_{t}(x, 0)=\omega_{1}, \\
\chi(x, \rho, 0)=\chi_{0}=h_{01}-h_{02} .
\end{gathered}
$$

Multiplying (47) by $\omega_{t}$ and integrating the result over $\Omega$, we get

$$
\begin{aligned}
& \frac{1}{2} \frac{d}{d t}\left(\left\|\omega_{t}(t)\right\|^{2}+\|\Delta \omega(t)\|^{2}\right. \\
& \left.\quad-\left(\int_{0}^{t} g(s) d s\right)\|\nabla \omega(t)\|^{2}+(g \circ \nabla \omega)(t)\right) \\
& \quad+\mu_{1}\left\|\omega_{t}(t)\right\|^{2} \\
& \leq\left|\left(M\left(\|\nabla u\|^{2}\right) \Delta u-M\left(\|\nabla v\|^{2}\right) \Delta v, \omega_{t}\right)\right| \\
& \quad+\mu_{2} \int_{\Omega}|\chi(x, 1, t)|\left|\omega_{t}\right| d x \\
& \quad+\int_{\Omega}|f(u)-f(v)|\left|\omega_{t}\right| d x
\end{aligned}
$$

By mean value theorem and Hölder's inequality, we derive

$$
\begin{aligned}
& \left|\left(M\left(\|\nabla u\|^{2}\right) \Delta u-M\left(\|\nabla v\|^{2}\right) \Delta v, \omega_{t}\right)\right| \\
& =\mid \int_{\Omega}\left(M\left(\|\nabla u\|^{2}\right) \Delta u-M\left(\|\nabla u\|^{2}\right) \Delta v\right. \\
& \left.+M\left(\|\nabla u\|^{2}\right) \Delta v-M\left(\|\nabla v\|^{2}\right) \Delta v\right) \omega_{t} d x \mid \\
& \leq \int_{\Omega} M\left(\|\nabla u\|^{2}\right)|\Delta \omega|\left|\omega_{t}\right| d x \\
& \quad+\int_{\Omega}\left|M^{\prime}(\eta)\right|\left|\left(\|\nabla u\|^{2}-\|\nabla v\|^{2}\right)\right||\Delta v|\left|\omega_{t}\right| d x \\
& \leq \int_{\Omega} M\left(\|\nabla u\|^{2}\right)|\Delta \omega|\left|\omega_{t}\right| d x \\
& \quad+C_{1}(|\nabla u|+|\nabla v|)|\nabla \omega||\Delta v|\left|\omega_{t}\right| d x \\
& \leq C_{1}\left(\|\Delta \omega\|^{2}+\left\|\omega_{t}\right\|^{2}\right) \\
& \quad+C_{1}\left(\|\nabla u\|_{L^{\infty}}+\|\nabla v\|_{L^{\infty}}\right)\|\nabla \omega\|_{L^{\infty}}\|\Delta v\|\left\|\omega_{t}\right\| \\
& \leq C_{1}\left(\|\Delta \omega\|^{2}+\left\|\omega_{t}\right\|^{2}\right) .
\end{aligned}
$$

It follows from (12)-(13) and Hölder's inequality that

$$
\begin{aligned}
\int_{\Omega} \mid f & (u)-f(v)|| \omega_{t} \mid d x \\
& \leq C_{1} \int_{\Omega}\left(1+|u|^{\rho}+|v|^{\rho}\right)|\omega|\left|\omega_{t}\right| d x \\
& \leq C_{1}\left(\|u\|_{2(\rho+1)}^{\rho}+\|v\|_{2(\rho+1)}^{\rho}\right)\|\omega\|_{2(\rho+1)}\left\|\omega_{t}\right\| \\
& \leq C_{1}\left(\|\Delta \omega\|^{2}+\left\|\omega_{t}\right\|^{2}\right) .
\end{aligned}
$$

Moreover,

$$
\begin{aligned}
& \mu_{2} \int_{\Omega}|\chi(x, 1, t)|\left|\omega_{t}\right| d x \\
& \quad \leq \frac{\mu_{2}}{2} \int_{\Omega} \chi^{2}(x, 1, t) d x+\frac{\mu_{2}}{2}\left\|\omega_{t}\right\|^{2} .
\end{aligned}
$$

Noting that (35)-(36) and combining (54)-(57), we conclude that

$$
\begin{aligned}
\frac{d}{d t} \mathscr{E}(t) & +\left(\mu_{1}-\frac{\xi}{2 \tau}-\frac{\mu_{2}}{2}\right)\left\|\omega_{t}\right\|^{2} \\
& +\left(\frac{\xi}{2 \tau}-\frac{\mu_{2}}{2}\right) \int_{\Omega} \chi^{2}(x, 1, t) d x \\
\leq & C_{1}\left(\|\Delta \omega\|^{2}+\left\|\omega_{t}\right\|^{2}\right),
\end{aligned}
$$

where

$$
\begin{aligned}
\mathscr{E}(t)= & \left\|\omega_{t}(t)\right\|^{2}+\|\Delta \omega(t)\|^{2}-\left(\int_{0}^{t} g(s) d s\right)\|\nabla \omega(t)\|^{2} \\
& +(g \circ \nabla \omega)(t)+\frac{\xi}{2} \int_{\Omega} \int_{0}^{1} \chi^{2}(x, \rho, t) d \rho d x .
\end{aligned}
$$

It follows (19) that

$$
\mathscr{E}(t) \leq \mathscr{E}(0)+C_{1} \int_{0}^{t}\left(\|\Delta \omega\|^{2}+\left\|\omega_{t}\right\|^{2}\right)(s) d s
$$

which, along with (9), gives

$$
\|\Delta \omega\|^{2}+\left\|\omega_{t}\right\|^{2} \leq \mathscr{E}(0)+C_{1} \int_{0}^{t}\left(\|\Delta \omega\|^{2}+\left\|\omega_{t}\right\|^{2}\right)(s) d s .
$$

Applying Gronwall's inequality to (61), we get

$$
\|\Delta \omega\|^{2}+\left\|\omega_{t}\right\|^{2} \leq e^{C_{1} t} \mathscr{E}(0) .
$$

This shows that solution of problem (17)-(18) depends continuously on the initial data. In particular, problem (17)-(18) has a unique stronger solution.

We can prove the continuous dependence and uniqueness for weak solutions by using density arguments (see, e.g., Cavalcanti et al. [27]) which also can be found in Lions [29] (Chapter 1, Theorem 1.2) by using a regularization method and in Pata and Zucchi [31] or Giorgi et al. [32] by using the mollifiers.

This ends the proof of Theorem 1. 


\section{General Decay}

In this section, we will establish the decay property of the solution for problem (17)-(18) in the case $\mu_{2}<\mu_{1}$. Motivated by $[27,33]$, we use a perturbed energy method and suitable Lyapunov functionals.

We first consider stronger solutions. Define the modified energy by

$$
\begin{aligned}
F(t)= & \frac{1}{2}\left\|u_{t}(t)\right\|^{2}+\frac{1}{2}\|\Delta u(t)\|^{2} \\
& +\frac{1}{2} \widehat{M}\left(\|\nabla u(t)\|^{2}\right)+\int_{\Omega} \widehat{f}(u(t)) d x \\
& -\frac{1}{2}\left(\int_{0}^{t} g(s) d s\right)\|\nabla u(t)\|^{2}+\frac{1}{2}(g \circ \nabla u)(t) \\
& +\frac{\xi}{2} \int_{\Omega} \int_{0}^{1} z^{2}(x, \rho, t) d \rho d x,
\end{aligned}
$$

where $\xi$ is a positive constant satisfying (19).

It follows from (9) and (14) that

$$
\begin{aligned}
F(t) & =E(t)-\frac{1}{2}\left(\int_{0}^{t} g(s) d s\right)\|\nabla u(t)\|^{2}+\frac{1}{2}(g \circ \nabla u)(t) \\
& \geq l E(t),
\end{aligned}
$$

that is,

$$
E(t) \leq \frac{1}{l} F(t) .
$$

Lemma 3. Under the assumptions in Theorem 2, the modified energy functional defined by (63) satisfies that there exists a constant $c>0$ such that, for any $t \geq 0$,

$$
\begin{aligned}
\frac{d}{d t} F(t) \leq & -c \int_{\Omega} u_{t}^{2}(t) d x-c \int_{\Omega} z^{2}(x, 1, t) d x \\
& +\frac{1}{2}\left(g^{\prime} \circ \nabla u\right)(t)-\frac{1}{2} g(t)\|\nabla u(t)\|^{2} .
\end{aligned}
$$

Proof. For the same argument as (41) in Section 3.2, we can easily get (66). Here we omit the detailed proof.

Now we define the following functional:

$$
\Phi(t)=\int_{\Omega} u_{t}(t) u(t) d x
$$

Then we have the following lemma.

Lemma 4. Under the assumptions in Theorem 2, the functional $\Phi(t)$ defined in (67) satisfies that, for any $\eta>0$,

$$
\begin{aligned}
\frac{d}{d t} \Phi(t) \leq & \left(1+\frac{\mu_{1}}{4 \eta}\right)\left\|u_{t}(t)\right\|^{2} \\
& -\left(l-\eta \lambda-2 \eta \lambda \lambda_{1}\left(\mu_{1}+\mu_{2}\right)\right)\|\Delta u(t)\|^{2} \\
& -\widehat{M}\left(\|\nabla u(t)\|^{2}\right)+\frac{\mu_{2}}{4 \eta} \int_{\Omega} z^{2}(x, 1, t) d x \\
& +\frac{1-l}{4 \eta \lambda}(g \circ \nabla u)(t),
\end{aligned}
$$

where $\lambda_{1}$ is the Poincaré constant.
Proof. By taking a derivative of (67) and using the first equation of (17), we conclude that

$$
\begin{aligned}
\frac{d}{d t} \Phi(t)= & \int_{\Omega} u_{t t}(t) u(t) d x+\left\|u_{t}(t)\right\|^{2} \\
= & \left\|u_{t}(t)\right\|^{2} \\
& +\int_{\Omega}\left(-\Delta^{2} u(t)+M\left(\|\nabla u(t)\|^{2}\right) \Delta u(t)\right. \\
& -\int_{0}^{t} g(t-s) \Delta u(s) d s \\
& \left.-\mu_{2} u_{t}(t)-\mu_{2} z(x, 1, t)-f(u)\right) \\
& \cdot u(t) d x \\
\leq & \left\|u_{t}(t)\right\|^{2}-\|\Delta u(t)\|^{2}-\widehat{M}\left(\|\nabla u(t)\|^{2}\right) \\
& +\int_{0}^{t} g(t-s)(\nabla u(s), \nabla u(t)) d s \\
& -\mu_{1} \int_{\Omega} u_{t}(t) u(t) d x-\mu_{2} \int_{\Omega} z(x, 1, t) u(t) d x \\
& -\int_{\Omega} f(u(t)) u(t) d x .
\end{aligned}
$$

Using Hölder's inequality, we know that, for any $\eta>0$,

$$
\begin{aligned}
& \int_{0}^{t} g(t-s)(\nabla u(s), \nabla u(t)) d s \\
&= \int_{0}^{t} g(t-s) \int_{\Omega}(\nabla u(s)-\nabla u(t)+\nabla u(t)) \cdot \nabla u(t) d x d s \\
& \leq \int_{0}^{t} g(t-s) \int_{\Omega}|\nabla u(t)-\nabla u(s)||\nabla u(t)| d x d s \\
&+\int_{0}^{t} g(s) d s \cdot\|\nabla u(t)\|^{2} \\
& \leq\|\nabla u(t)\|^{2} \int_{0}^{t} g(t-s)\|\nabla u(t)-\nabla u(s)\|^{2} d s \\
& \quad+\int_{0}^{t} g(s) d s \cdot\|\nabla u(t)\|^{2} \\
& \leq \eta\|\nabla u(t)\|^{2}+\frac{1}{4 \eta}\|g(t)\|_{L^{1}\left(\mathbb{R}^{+}\right)}(g \circ \nabla u)(t) \\
& \quad+\int_{0}^{t} g(s) d s \cdot\|\nabla u(t)\|^{2} \\
& \leq \eta \lambda\|\Delta u(t)\|^{2}+\frac{1}{4 \eta}\|g(t)\|_{L^{1}\left(\mathbb{R}^{+}\right)}(g \circ \nabla u)(t) \\
& \quad+\int_{0}^{t} g(s) d s \cdot\|\nabla u(t)\|^{2}
\end{aligned}
$$




$$
\begin{aligned}
\leq & (1-l+\eta \lambda)\|\Delta u(t)\|^{2} \\
& +\frac{1}{4 \eta}\|g(t)\|_{L^{1}\left(\mathbb{R}^{+}\right)}(g \circ \nabla u)(t) .
\end{aligned}
$$

By using Young's inequality and Poincaré's inequality and noting $\|\nabla u\|^{2} \leq \lambda\|\Delta u\|^{2}$, we infer that, for any $\eta>0$,

$$
\begin{gathered}
\int_{\Omega} u_{t}(t) u(t) d x \leq \eta \lambda \lambda_{1}\|\Delta u(t)\|^{2}+\frac{1}{4 \eta}\left\|u_{t}(t)\right\|^{2} \\
\int_{\Omega} z(x, 1, t) u(t) d x \\
\quad \leq \eta \lambda \lambda_{1}\|\Delta u(t)\|^{2}+\frac{1}{4 \eta} \int_{\Omega} z^{2}(x, 1, t) d x
\end{gathered}
$$

Combining (69)-(72) and noting (14), we complete the proof.

In order to handle the term $z(x, \rho, t)$, we introduce the functional

$$
\Psi(t):=\int_{\Omega} \int_{0}^{1} e^{-2 \tau \rho} z^{2}(x, \rho, t) d \rho d x .
$$

Then we have the following estimate.

Lemma 5. Under the assumptions in Theorem 2, the functional $\Psi(t)$ defined in (73) satisfies that

$$
\begin{aligned}
\frac{d}{d t} \Psi(t) \leq & -\rho \Psi(t)-\frac{c_{1}}{2 \tau} \int_{0}^{1} z^{2}(x, 1, t) d x \\
& +\frac{1}{2 \tau} \int_{0}^{1} u_{t}^{2}(t) d x,
\end{aligned}
$$

where $c_{1}$ is a positive constant.

Proof. Differentiating (73) with respect to $t$ and using the second equation (17), we obtain

$$
\begin{aligned}
\frac{d}{d t} \Psi(t)= & -\frac{1}{\tau} \int_{\Omega} \int_{0}^{1} e^{-2 \tau \rho} z(x, \rho, t) z_{\rho}(x, \rho, t) d \rho d x \\
= & -\int_{\Omega} \int_{0}^{1} e^{-2 \tau \rho} z^{2}(x, \rho, t) d \rho d x \\
& -\frac{1}{2 \tau} \int_{\Omega} \int_{0}^{1} \frac{\partial}{\partial \rho}\left(e^{-2 \tau \rho} z^{2}(x, \rho, t)\right) d \rho d x
\end{aligned}
$$

Then it is easy to verify that there exists a constant $c_{1}>0$ satisfying (74).

Proof of Theorem 2. We define the Lyapunov functional

$$
\mathscr{G}(t):=F(t)+\epsilon \Phi(t)+\epsilon \Psi(t),
$$

where $\epsilon>0$ is a real number which will be taken later.

First, we claim that there exist two positive constants $\beta_{1}$ and $\beta_{2}$ such that, for any $t \geq 0$,

$$
\beta_{1} F(t) \leq \mathscr{G}(t) \leq \beta_{2} F(t) .
$$

Indeed, it is easy to get

$$
\begin{aligned}
& |\Phi(t)+\Psi(t)| \\
& \quad \leq \frac{1}{2}\left\|u_{t}(t)\right\|^{2}+\frac{1}{2 \lambda^{\prime}}\|\Delta u(t)\|^{2}+\int_{\Omega} \int_{0}^{1} z^{2}(x, \rho, t) d \rho d x \\
& \quad \leq \frac{1}{l} \max \left\{1, \frac{1}{\lambda^{\prime}}, \xi\right\} F(t),
\end{aligned}
$$

where $\lambda^{\prime}>0$ is the first eigenvalue of $\Delta^{2} u=\lambda u$ in $\Omega$ with $u=\Delta u=0$ on $\partial \Omega$. Choosing $C_{1}=(1 / l) \max \left\{1,1 / \lambda^{\prime}, \xi\right\}$, we know that

$$
|\mathscr{G}(t)-F(t)|=\epsilon|\Phi(t)+\Psi(t)| \leq \epsilon C_{1} F(t) .
$$

Now putting $\epsilon>0$ small enough and choosing $\beta_{1}:=1-\epsilon C_{1}>$ 0 and $\beta_{2}=1+\epsilon C_{1}>0$, we see that (77) holds.

Next, combining (66), (68), and (74), we arrive at

$$
\begin{aligned}
\frac{d}{d t} \mathscr{G}(t) \leq & -\left(c-\epsilon\left(1+\frac{\mu_{1}}{4 \eta}\right)-\frac{\epsilon}{2 \tau}\right)\left\|u_{t}(t)\right\|^{2} \\
& -\epsilon \widehat{M}\left(\|\nabla u(t)\|^{2}\right)-\rho \epsilon \Psi(t) \\
& -\epsilon\left(l-\eta \lambda-2 \eta \lambda \lambda_{1}\left(\mu_{1}+\mu_{2}\right)\right)\|\Delta u(t)\|^{2} \\
& -\left(c+\frac{\epsilon c_{1}}{2 \tau}-\frac{\epsilon}{4 \eta}\right) \int_{\Omega} z^{2}(x, 1, t) d x \\
& +\frac{1-l}{4 \eta \epsilon}(g \circ \nabla u)(t)+\frac{1}{2}\left(g^{\prime} \circ \nabla u\right)(t) \\
& -\frac{1}{2} g(t)\|\nabla u(t)\|^{2} .
\end{aligned}
$$

Now we choose $\eta>0$ and $\epsilon>0$ so small that we can take two positive constants $\alpha_{1}$ and $\alpha_{2}$ such that, for any $t \geq 0$,

$$
\mathscr{G}^{\prime}(t) \leq-\alpha_{1} F(t)+\alpha_{2}(g \circ \nabla u)(t) .
$$

Multiplying (81) by $\mu(t)$, we have, for any $t \geq 0$,

$$
\mu(t) \mathscr{G}^{\prime}(t) \leq-\alpha_{1} \mu(t) F(t)+\alpha_{2} \mu(t)(g \circ \nabla u)(t),
$$

which, along with (10) and (66), implies

$$
\begin{aligned}
\mu(t) \mathscr{G}^{\prime}(t) & \leq-\alpha_{1} \mu(t) F(t)-\alpha_{2}\left(g^{\prime} \circ \nabla u\right)(t) \\
& \leq-\alpha_{1} \mu(t) F(t)-2 \alpha_{2} E^{\prime}(t), \quad \forall t \geq 0
\end{aligned}
$$

that is,

$$
\begin{gathered}
\left(\mu(t) \mathscr{G}(t)+2 \alpha_{2} F(t)\right)^{\prime}-\mu^{\prime}(t) \mathscr{G}(t) \\
\leq-\alpha_{1} \mu(t) F(t), \quad \forall t \geq 0 .
\end{gathered}
$$

Denote $\mathscr{F}(t)=\mu(t) \mathscr{G}(t)+2 \alpha_{2} F(t)$, and then $\mathscr{F}(t)$ is equivalent to $F(t)$; that is,

$$
\mathscr{F}(t) \sim F(t) .
$$


Thus we conclude that, for any $t \geq 0$,

$$
\mathscr{F}^{\prime}(t) \leq-\alpha_{2} \mu(t) F(t) \leq-\alpha_{3} \mu(t) \mathscr{F}(t) .
$$

Integrating $(86)$ over $(0, t)$, we will see the following:

$$
\mathscr{F}(t) \leq \mathscr{F}(0) \exp \left(-\alpha_{3} \int_{0}^{t} \mu(s) d s\right), \quad \forall t \geq 0,
$$

which, together with (65), (77), and (85), gives (25).

This proves the general decay for regular solutions. We can extend the result to weak solutions by using a standard density argument; one can refer to Cavalcanti et al. [27]. The proof is hence complete.

Remark 6. There are some open problems concerning our present work, and here we give some of them.

(1) It is obvious that the weak damping term $\mu_{1} u_{t}$ plays a crucial role in our proofs. It is still an open problem when $\mu_{1}=0$.

(2) We only obtain the general decay for $\mu_{1}>\mu_{2}$. Whether the stability property holds for $\mu_{1}=\mu_{2}$ is still open.

(3) It is interesting to study that the weight of the delay is bigger than the weight of the damping; that is, $\mu_{1}<\mu_{2}$.

\section{Conflict of Interests}

The author declares that there is no conflict of interests regarding the publication of this paper.

\section{Acknowledgments}

The author would like to thank the referees for their helpful comments. This work was supported by the Fundamental Research Funds for the Central Universities with Contract no. JBK150128.

\section{References}

[1] S. Woinowsky-Krieger, "The effect of axial force on the vibration of hinged bars," Journal of Applied Mechanics, vol. 17, pp. 35-36, 1950.

[2] E. H. de Brito, "Decay estimates for the generalized damped extensible string and beam equations," Nonlinear Analysis: Theory, Methods \& Applications, vol. 8, no. 12, pp. 1489-1496, 1984.

[3] M. M. Cavalcanti, V. N. D. Cavalcanti, and J. A. Soriano, "Global existence and asymptotic stability for the nonlinear and generalized damped extensible plate equation," Communications in Contemporary Mathematics, vol. 6, no. 5, pp. 705-731, 2004.

[4] M. M. Cavalcanti, V. N. Domingos Cavalcanti, and J. A. Soriano, "Global existence and uniform decay rates to the KirchhoffCarrier equation with nonlinear dissipation," Advances in Differential Equations, vol. 6, no. 6, pp. 85-116, 2001.

[5] T. F. Ma, "Boundary stabilization for a non-linear beam on elastic bearings," Mathematical Methods in the Applied Sciences, vol. 24, no. 8, pp. 583-594, 2001.
[6] T. F. Ma and V. Narciso, "Global attractor for a model of extensible beam with nonlinear damping and source terms," Nonlinear Analysis: Theory, Methods \& Applications, vol. 73, no. 10, pp. 3402-3412, 2010.

[7] M. de Lacerda Oliveira and O. A. de Lima, "Exponential decay of the solutions of the beams system," Nonlinear Analysis, Theory, Methods \& Applications, vol. 42, no. 7, pp. 1271-1291, 2000.

[8] J. Y. Park and S. H. Park, "General decay for a nonlinear beam equation with weak dissipation," Journal of Mathematical Physics, vol. 51, no. 7, Article ID 073508, 2010.

[9] S. K. Patcheu, "On a global solution and asymptotic behavior for the generalized damped extensible beam equation," Journal of Differential Equations, vol. 135, no. 2, pp. 299-314, 1997.

[10] J. E. M. Rivera and L. H. Fatori, "Smoothing effect and propagations of singularities for viscoelastic plates," Journal of Mathematical Analysis and Applications, vol. 206, no. 2, pp. 397427, 1997.

[11] J. E. M. Rivera, E. C. Lapa, and R. Barreto, "Decay rates for viscoelastic plates with memory," Journal of Elasticity, vol. 44, no. 1, pp. 61-87, 1996.

[12] M. Tusnal, "Semi-internal stabilization for a nonlinear EulerBernoulli equation," Mathematical Methods in the Applied Sciences, vol. 19, pp. 897-907, 1996.

[13] C. F. Vasconcellos and L. M. Teixeira, "Existence, uniqueness and stabilization for a nonlinear plate system with nonlinear damping," Annales de la Faculté des Sciences de Toulouse Mathématiques, vol. 8, no. 1, pp. 173-193, 1999.

[14] Z. Yang, "Longtime behavior for a nonlinear wave equation arising in elasto-plastic flow," Mathematical Methods in the Applied Sciences, vol. 32, no. 9, pp. 1082-1104, 2009.

[15] Z. Yang, "On an extensible beam equation with nonlinear damping and source terms," Journal of Differential Equations, vol. 254, no. 9, pp. 3903-3927, 2013.

[16] D. Andrade, M. A. Jorge Silva, and T. F. Ma, "Exponential stability for a plate equation with $p$-Laplacian and memory terms," Mathematical Methods in the Applied Sciences, vol. 35, no. 4, pp. 417-426, 2012.

[17] S. Berrimi and S. A. Messaoudi, "Existence and decay of solutions of a viscoelastic equation with a nonlinear source," Nonlinear Analysis: Theory, Methods \& Applications, vol. 64, no. 10, pp. 2314-2331, 2006.

[18] S. A. Messaoudi, "General decay of the solution energy in a viscoelastic equation with a nonlinear source," Nonlinear Analysis, Theory, Methods \& Applications, vol. 69, no. 8, pp. 2589-2598, 2008.

[19] S. A. Messaoudi and N.-E. Tartar, "Global existence and asymptotic behavior for a nonlinear viscoelastic equation," Mathematical Sciences Research Journal, vol. 7, no. 4, pp. 136149, 2003.

[20] S. A. Messaoudi and N.-E. Tatar, "Exponential and polynomial decay for a quasilinear viscoelastic equation," Nonlinear Analysis: Theory, Methods \& Applications, vol. 68, no. 4, pp. 785-793, 2008.

[21] R. Datko, J. Lagnese, and M. P. Polis, "An example on the effect of time delays in boundary feedback stabilization of wave equations," SIAM Journal on Control and Optimization, vol. 24, no. 1, pp. 152-156, 1986.

[22] G. Q. Xu, S. P. Yung, and L. K. Li, "Stabilization of wave systems with input delay in the boundary control," ESAIM: Control, Optimisation and Calculus of Variations, vol. 12, no. 4, pp. 770785, 2006. 
[23] M. Kirane and B. Said-Houari, "Existence and asymptotic stability of a viscoelastic wave equation with a delay," Zeitschrift fur Angewandte Mathematik und Physik, vol. 62, no. 6, pp. 10651082, 2011.

[24] S. Nicaise and C. Pignotti, "Stabilization of the wave equation with boundary or internal distributed delay," Differential and Integral Equations, vol. 21, pp. 935-958, 2008.

[25] S. Nicaise, J. Valein, and E. Fridman, "Stability of the heat and of the wave equations with boundary time-varying delays," Discrete and Continuous Dynamical Systems_-Series S, vol. 2, no. 3, pp. 559-581, 2009.

[26] S. Nicaise and J. Valein, "Stabilization of second order evolution equations with unbounded feedback with delay," ESAIM: Control, Optimisation and Calculus of Variations, vol. 16, no. 2, pp. 420-456, 2010.

[27] M. M. Cavalcanti, V. N. Domingos Cavalcanti, and T. F. Ma, "Exponential decay of the viscoelastic Euler-Bernoulli equation with a nonlocal dissipation in general domains," Differential and Integral Equations, vol. 17, pp. 495-510, 2004.

[28] M. A. Jorge Silva and T. F. Ma, "On a viscoelastic plate equation with history setting and perturbation of $p$-Laplacian type," IMA Journal of Applied Mathematics, vol. 78, no. 6, pp. 1130-1146, 2013.

[29] J. L. Lions, Quelques Méthodes de Résolution des Problèmes aux Limites Non Linéaires, Dunod Gauthier-Villars, Paris, France, 1969.

[30] J. U. Kim, "A boundary thin obstacle problem for a wave equation," Communications in Partial Differential Equations, vol. 14, no. 8-9, pp. 1011-1026, 1989.

[31] V. Pata and A. Zucchi, "Attractors for a damped hyperbolic equation with linear memory," Advances in Mathematical Sciences and Applications, vol. 11, pp. 505-529, 2001.

[32] C. Giorgi, V. Pata, and A. Marzocchi, "Asymptotic behavior of a semilinear problem in heat conduction with memory," Nonlinear Differential Equations and Applications, vol. 5, no. 3, pp. 333-354, 1998.

[33] E. Zuazua, "Exponential decay for the semilinear wave equation with locally distributed damping," Communications in Partial Differential Equations, vol. 15, no. 2, pp. 205-235, 1990. 


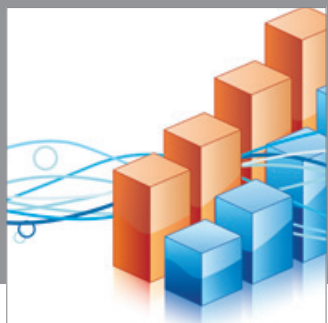

Advances in

Operations Research

mansans

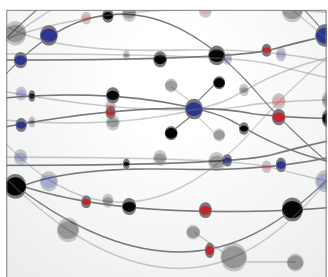

The Scientific World Journal
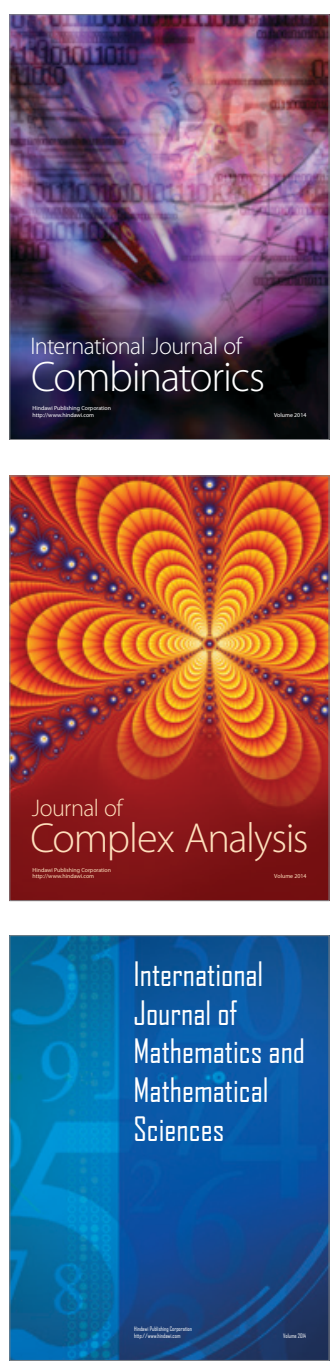
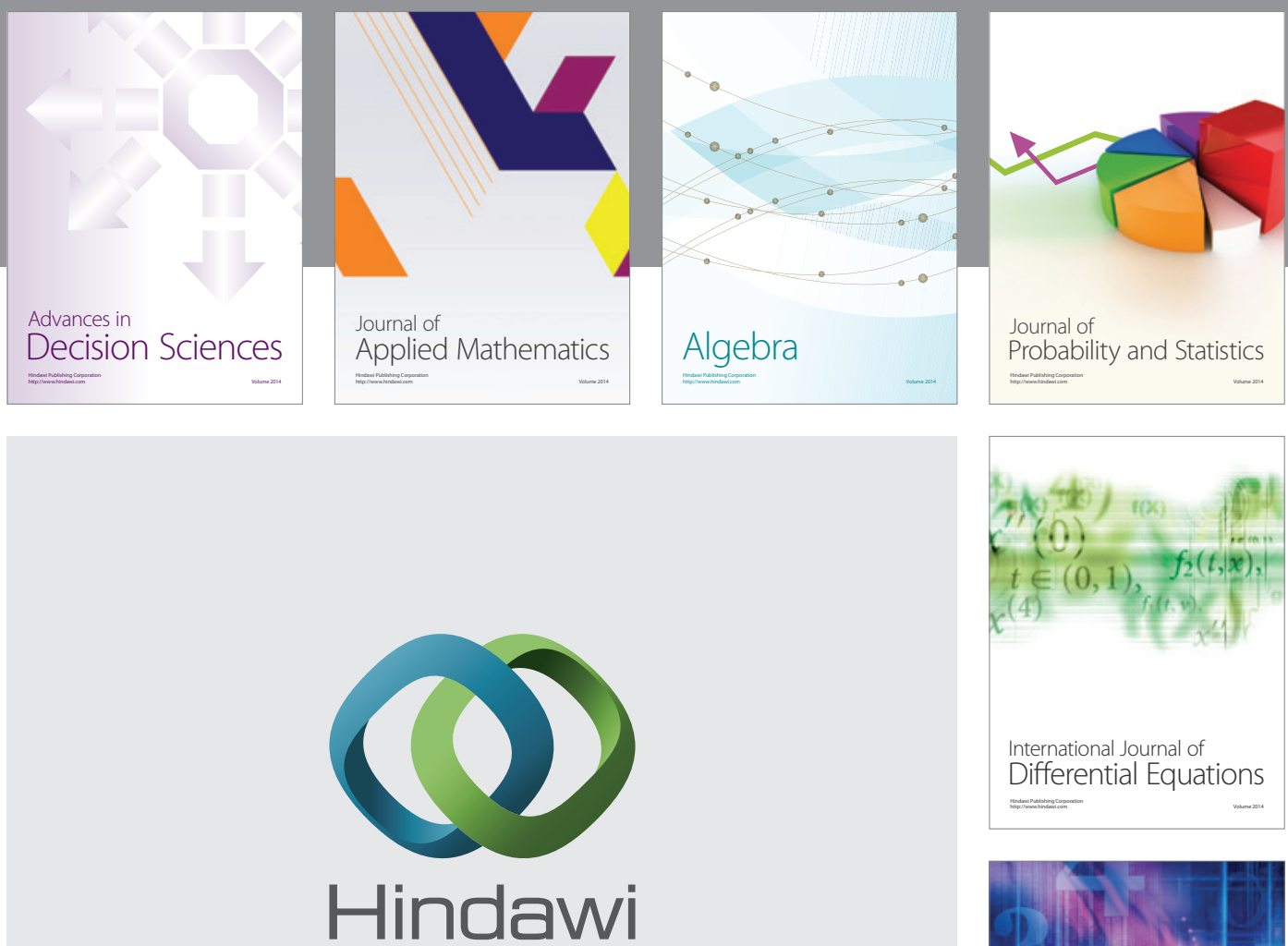

Submit your manuscripts at http://www.hindawi.com
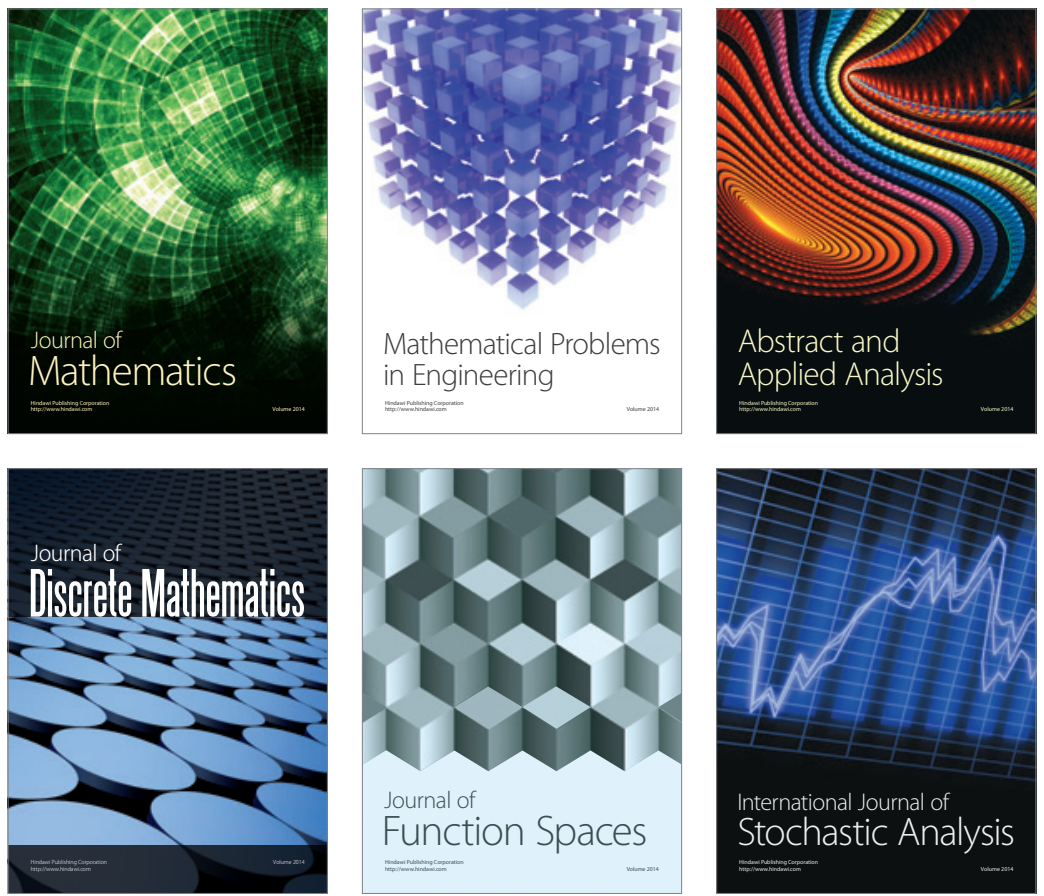

Journal of

Function Spaces

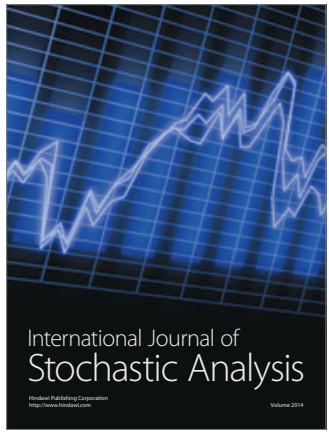

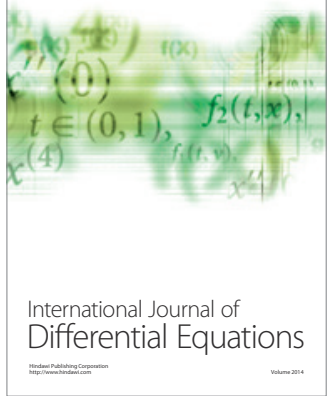
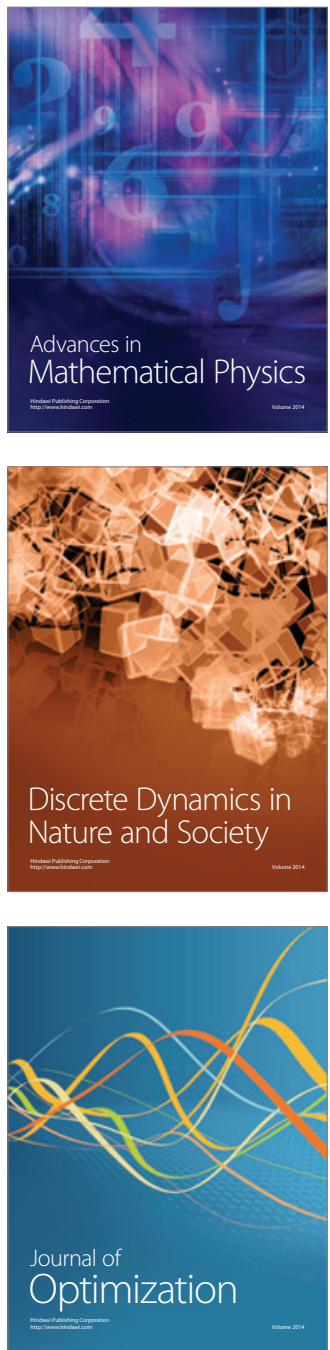